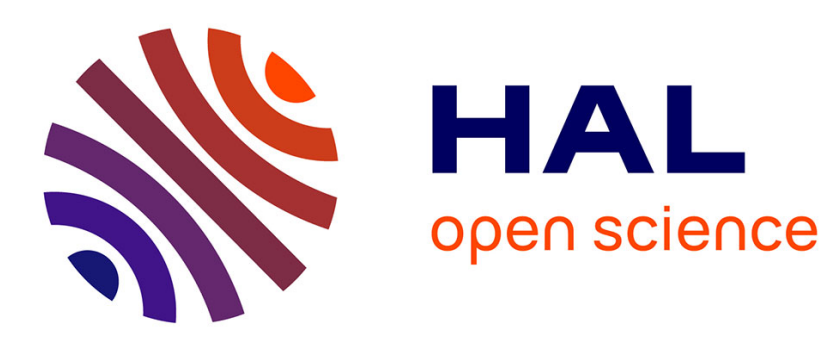

\title{
Stochastic stability of Markov jump hyperbolic systems with application to traffic flow control
}

Liguo Zhang, Christophe Prieur

\section{To cite this version:}

Liguo Zhang, Christophe Prieur. Stochastic stability of Markov jump hyperbolic systems with application to traffic flow control. Automatica, 2017, 86, pp.29-37. 10.1016/j.automatica.2017.08.007 . hal-01585772

\section{HAL Id: hal-01585772 \\ https://hal.science/hal-01585772}

Submitted on 12 Sep 2017

HAL is a multi-disciplinary open access archive for the deposit and dissemination of scientific research documents, whether they are published or not. The documents may come from teaching and research institutions in France or abroad, or from public or private research centers.
L'archive ouverte pluridisciplinaire $\mathbf{H A L}$, est destinée au dépôt et à la diffusion de documents scientifiques de niveau recherche, publiés ou non, émanant des établissements d'enseignement et de recherche français ou étrangers, des laboratoires publics ou privés. 


\title{
Stochastic Stability of Markov Jump Hyperbolic Systems with Application to Traffic Flow Control ${ }^{1}$
}

\author{
Liguo Zhang ${ }^{\mathrm{a}, \mathrm{b}}$, Christophe Prieur ${ }^{\mathrm{c}}$ \\ ${ }^{a}$ School of Electronic Information and Control Engineering, Beijing University of Technology, 100124, Beijing, China. \\ ${ }^{\mathrm{b}}$ Key Laboratory of Computational Intelligence and Intelligent Systems, 100124, Beijing, China. \\ ${ }^{\mathrm{c}}$ Univ. Grenoble Alpes, CNRS, Gipsa-lab, F-38000 Grenoble, France.
}

\begin{abstract}
In this paper, we investigate the stochastic stability of linear hyperbolic conservation laws governed by a finite-state Markov chain. Both system matrices and boundary conditions are subject to the Markov switching. The existence and uniqueness of weak solutions are developed for the stochastic hyperbolic initial boundary value problem. By means of Lyapunov techniques some sufficient conditions are obtained by seeking the balance between the boundary condition and the transition probability of the Markov process. Particularly, boundary feedback control of a stochastic traffic flow model is developed for the freeway transportation system by integrating the on-ramp metering with the speed limit control.
\end{abstract}

Key words: Stochastic hyperbolic conservation laws, Markov process, Stochastic stability, Lyapunov function, Traffic flow control.

\section{Introduction}

Many physical or engineering processes may be represented by the hyperbolic partial differential equations (PDEs) of conservation laws in one space dimension, such as Saint-Venant equation for open channels (de Halleux et al. (2003)), Euler equation for gas pipes (Gugat et al. (2011)), and Aw-Rascle equation for road traffic (Aw and Rascle (2000)). In such systems, the system matrices and the boundary conditions can both be subject to abrupt changes in their structures and parameters induced by the external causes or the internal mechanism. For example, in the freeway transportation systems, it can be the phase transition of traffic modes Colombo (2003)), or the random flux at the boundaries (Haut et al. (2007)). In such situations, it is more realistic to model the dynamic behaviors of these processes with switched hyperbolic systems.

Many results have been made for boundary stability of switched hyperbolic systems. In Amin et al. (2012), the exponential stability is given under arbitrary switching

1 This work is supported by the National Natural Science Foundation of China (NSFC, grant No. 61374076, 61533002) and the International Cooperation and Exchange Program of NSFC (grant No. 61111130119). using the propagation of solutions along the characteristics. In Prieur et al. (2014), using Lyapunov techniques some sufficient conditions are obtained for the exponential stability uniformly. Switching boundary control for semilinear hyperbolic balance equations is considered in Hante et al. (2009). In Lamare et al. (2015), stabilizing switching controllers are developed based on the steepest decent selection of the Lyapunov function.

In this paper, we consider a class of switched hyperbolic systems, named the Markov jump linear hyperbolic (MJLH) systems, in which mode switching is governed by a Markov chain and all modes are linear hyperbolic conservation laws. The boundary stabilization for hyperbolic systems (Li (1994); Coron et al. (2007)) and the stochastic stability for the Markov jump linear (MJL) systems of continuous-time (Costa et al. (2013)) or discrete-time cases (Boukas (2005)) have been studied for many years independently. The main contribution of this work is that the boundary stochastic stability for the MJLH systems is firstly obtained by means of Lyapunov techniques. The matrix inequality condition is based on the balance between the boundary condition of the hyperbolic conservation laws and the transition probability of the Markov process.

A second contribution of our work is the application to 
boundary control of freeway traffic. Due to the existence of a large number of uncertainties, such as demand variability, capacity decrease, etc., the local freeway traffic may randomly lie in the free-flow mode or in the congestion mode (Boel and Mihaylova (2006); Sumalee et al. (2011)). We develop a two-mode MJLH model to represent the quasilinear Aw-Rascle equation and design boundary feedback strategies by integrating the onramp metering with the speed limiting control. Theoretical contributions guarantee the stochastic exponential convergence of the MJLH traffic flow model, even with different transition probabilities of the Markov chain.

This paper is organized as follows. The class of MJLH systems and the wellposedness of weak solutions are given in Section 2. In Section 3, the main result on the sufficient conditions of the exponentially mean-square stable are derived for MJLH systems. Numerical computation of the conditions is discussed in Section 4. Finally, in Section 5, as a matter of illustration, an application of boundary feedback control of freeway traffic based on the MJLH traffic flow model is presented.

Notation: $\mathbb{R}_{+}, \mathbb{R}^{n}, \mathbb{R}^{n \times n}$ are the sets of non-negative reals, $n$-order vectors and matrices, respectively. The set of diagonal positive matrices in $\mathbb{R}^{n \times n}$ is denoted by $\mathcal{D}_{+}^{n}$. Given a matrix $A$, the transpose matrix is denoted as $A^{\top}, \lambda_{\max }(A), \rho(A)$ are the largest real parts of all eigenvalues and the spectral radius of $A$. $A<(\leq) B$ denotes $B-A$ is a positive definite (semidefinite) matrix. Given two real values $t_{1}$ and $t_{2}, t_{1} \wedge t_{2}$ denotes the minimal value between $t_{1}$ and $t_{2}$. The Euclidean norm in $\mathbb{R}^{n}$ is denoted by $|\cdot|$ and the associated matrix norm is $\|\cdot\|$. Given a function $g:[0,1] \rightarrow \mathbb{R}^{n}$, its $L^{2}$-norm is $\|g\|_{L^{2}(0,1)}=\sqrt{\int_{0}^{1}|g(x)|^{2} d x}$. We call $L^{2}(0,1)$ the space of all measurable functions $g(x)$ for which $\|g\|_{L^{2}(0,1)}<\infty$.

\section{Markov Jump Linear Hyperbolic Systems}

Let $(\Omega, \mathcal{F}, \operatorname{Pr})$ be a complete probability space equipped with a filtration $\left\{\mathcal{F}_{t} ; t \in \mathbb{R}_{+}\right\}$satisfying the usual hypotheses, that is, a right-continuous filtration augmented by all null sets in the Pr-completion of $\mathcal{F}$.

We consider a homogeneous Markov process $\{\sigma(t) ; t \in$ $\left.\mathbb{R}_{+}\right\}$adapted to the filtration $\left\{\mathcal{F}_{t} ; t \in \mathbb{R}_{+}\right\}$, with rightcontinuous trajectories and taking values on the set $\mathcal{S}=$ $\{1,2, \ldots, N\}$, where $N$ is a positive integer number. The infinitesimal generator $\Pi \in \mathbb{R}^{N \times N}$ of Markov process $\sigma(t)$ is given by

$$
\begin{aligned}
\operatorname{Pr}\{\sigma(t+\Delta t) & =j \mid \sigma(t)=i\} \\
& = \begin{cases}\pi_{i j} \Delta t+o(\Delta t), & \text { if } i \neq j \\
1+\pi_{i i} \Delta t+o(\Delta t), & \text { if } i=j\end{cases}
\end{aligned}
$$

where $\Delta t>0$ is constant (it is seen as a small time increment) and $o(\cdot)$ is a function satisfying $\lim _{\Delta t \rightarrow 0} \frac{o(\Delta t)}{\Delta t}=0$. Here $\pi_{i j} \geq 0$, for $i \neq j$, is the transition rate from mode $i$ at time $t$ to mode $j$ at time $t+\Delta t$, while

$$
\pi_{i i}=-\sum_{j=1, j \neq i}^{N} \pi_{i j}
$$

Let $\left\{\tau_{k} ; k=0,1, \ldots\right\}$ be the successive sojourn times between jumps, then $t_{k}=\sum_{l=0}^{k-1} \tau_{l}$, for $k=1,2, \ldots$, be the waiting time for the $k$ th jump with $t_{0}=0$.

Stating in mode $\sigma(0)=i$, the process sojourns there for a duration of time that is exponentially distributed with parameter $-\pi_{i i}$. The process then jumps to mode $j \neq i$ with probability $-\frac{\pi_{i j}}{\pi_{i i}}$, and the sojourn time in mode $j$ is exponentially distributed with parameter $-\pi_{j j}$, and so on. We further assume that the Markov process is irreducible. Under this condition, $\sigma(t)$ has a unique stationary probability distribution $\gamma=\left[\gamma_{1} \ldots \gamma_{N}\right]^{\top}$, which can be determined by solving the following linear equation $\gamma^{\top} \Pi=0$ subject to $\sum_{j=1}^{N} \gamma_{j}=1$ and $\gamma_{j}>0$, for all $j \in \mathcal{S}$ (Costa et al. 2013, Definition 2.9).

We consider the following Markov jump linear hyperbolic (MJLH for short) conservation laws of the form

$$
\partial_{t} \xi(x, t)+\Lambda_{\sigma(t)} \partial_{x} \xi(x, t)=0,
$$

where $t \in \mathbb{R}_{+}, x \in[0,1], \xi:[0,1] \times \mathbb{R}_{+} \rightarrow \mathbb{R}^{n}$ is the system state, the Markov process $\sigma(t): \mathbb{R}_{+} \rightarrow \mathcal{S}$ is a stochastic switching signal deciding the current operation mode. For all $i \in \mathcal{S}$, the system matrix $\Lambda_{i} \in \mathbb{R}^{n \times n}$ is a diagonal matrix with non-zero diagonal entries such that

$$
\Lambda_{i}=\operatorname{diag}\left\{\lambda_{1}^{i}, \lambda_{2}^{i}, \cdots, \lambda_{n}^{i}\right\},
$$

with $\lambda_{j}^{i}<0$ for $j \in\left\{1, \ldots, m_{i}\right\}$ and $\lambda_{j}^{i}>0$ for the other $j \in\left\{m_{i}+1, \ldots, n\right\}$.

According to the sign of each characteristic velocity $\lambda_{j}^{i}$, $j=\{1, \ldots, n\}, i \in \mathcal{S}$, we introduce the notation $\xi_{-}^{i}(\cdot)=$ $\left[\xi_{1}(\cdot), \cdots, \xi_{m_{i}}(\cdot)\right]^{\top}, \xi_{+}^{i}(\cdot)=\left[\xi_{m_{i}+1}(\cdot), \cdots, \xi_{n}(\cdot)\right]^{\top}$, and thus $\xi=\left[\xi_{-}^{i}, \xi_{+}^{i}\right]^{\top}$, for all $i \in \mathcal{S}$.

For MJLH system (2), the boundary condition also is a stochastic process, corresponding to the Markov chain $\sigma(t)$, written as

$$
\left[\begin{array}{l}
\xi_{-}^{\sigma(t)}(1, t) \\
\xi_{+}^{\sigma(t)}(0, t)
\end{array}\right]=G_{\sigma(t)}\left[\begin{array}{l}
\xi_{-}^{\sigma(t)}(0, t) \\
\xi_{+}^{\sigma(t)}(1, t)
\end{array}\right]
$$

where $G_{i}$ is a matrix in $\mathbb{R}^{n \times n}, i \in \mathcal{S}$. Let us introduce the matrices $G_{--}^{i}$ in $\mathbb{R}^{m_{i} \times m_{i}}, G_{-+}^{i}$ in $\mathbb{R}^{m_{i} \times\left(n-m_{i}\right)}, G_{+-}^{i}$ in $\mathbb{R}^{\left(n-m_{i}\right) \times m_{i}}$, and $G_{++}^{i}$ in $\mathbb{R}^{\left(n-m_{i}\right) \times\left(n-m_{i}\right)}$ such that $G_{i}=\left[\begin{array}{ll}G_{--}^{i} & G_{-+}^{i} \\ G_{+-}^{i} & G_{++}^{i}\end{array}\right]$. 
We consider the initial condition given by

$$
\xi(x, 0)=\xi^{0}(x), x \in(0,1),
$$

for a given function $\xi^{0}(\cdot) \in L^{2}(0,1)$ and a initial operation mode $\sigma(0) \in \mathcal{S}$.

For each mode $i \in \mathcal{S}$, as respective hyperbolic equation (2)-(4) holds a sojourn for a duration of time $\left\{\tau_{k} ; k=\right.$ $0,1, \ldots\}$, the existence and uniqueness of solution in the set $C^{0}\left([0, \infty), H^{1}(0,1)\right) \cap C^{1}\left([0, \infty), L^{2}(0,1)\right)$ with initial condition in $L^{2}(0,1)$ is quite classical, see e.g. (Bastin and Coron, 2016, Theorem A.4). Recently, the notion of solutions for an initial-boundary value problem of switched hyperbolic systems has been developed within the usual Lebesgue almost everywhere equivalence class, see e.g. Amin et al. (2012), and (Prieur et al. 2014, Proposition 3.1).

We now provide an existence and uniqueness result for the solutions of the MJLH system (2)-(4).

Proposition 1 The MJLH system (2)-(3) admits a unique solution $\xi=\xi(\cdot, t), t \in \mathbb{R}_{+}$, such that $E\left\{\|\xi(\cdot, t)\|_{L^{2}(0,1)}\right\}<\infty$, for any initial condition $\xi^{0} \in L^{2}(0,1)$ and any initial operation mode $\sigma(0) \in \mathcal{S}$, where $E\{\cdot\}$ stands for the mathematical expectation.

Proof. Recall that almost every sample path of stochastic process $\sigma(t), t \geq 0$, is a right-continuous step function with a finite number of jumps in any finite time interval. Then there exists a sequence $\left\{t_{k} ; k=0,1, \ldots\right\}$ of stopping times such that $t_{0}=0, \lim _{k \rightarrow \infty} t_{k}=\infty$, and $\sigma(t)=\sigma\left(t_{k}\right)$ on $t_{k} \leq t<t_{k+1}$ for any $k \geq 0$.

We then build iteratively the solution between successive stopping times. Let $T \in \mathbb{R}_{+}$be arbitrary, we first consider the MJLH system (2)-(3) on the time interval $t \in\left[0, t_{1} \wedge T\right]$ which becomes

$$
\partial_{t} \xi(x, t)+\Lambda_{\sigma(0)} \partial_{x} \xi(x, t)=0,
$$

for all $x \in(0,1)$ with the boundary condition of the form

$$
\left[\begin{array}{l}
\xi_{-}^{\sigma(0)}(1, t) \\
\xi_{+}^{\sigma(0)}(0, t)
\end{array}\right]=G_{\sigma(0)}\left[\begin{array}{l}
\xi_{-}^{\sigma(0)}(0, t) \\
\xi_{+}^{\sigma(0)}(1, t)
\end{array}\right],
$$

and the initial condition $\xi^{0} \in L^{2}(0,1)$. For any initial mode $\sigma(0) \in \mathcal{S}$, by (Bastin and Coron, 2016, Theorem A.4), the initial-boundary value problem (5)-(6) has a unique solution $\xi(\cdot, t) \in L^{2}(0,1)$, which satisfies

$$
\|\xi(\cdot, t)\|_{L^{2}(0,1)} \leq C_{1}\left\|\xi^{0}(\cdot)\right\|_{L^{2}(0,1)},
$$

for all $t \in\left[0, t_{1} \wedge T\right]$ with $C_{1}>0$, then almost surely we have

$$
E\left\{\left\|\xi\left(\cdot, t_{1} \wedge T\right)\right\|_{L^{2}(0,1)}\right\}<\infty .
$$

Setting $\xi^{1}(\cdot)=\xi\left(\cdot, \tau_{1} \wedge T\right)$, we next consider the MJLH system (2)-(3) on the time interval $t \in\left[t_{1} \wedge T, t_{2} \wedge T\right]$, which becomes

$$
\partial_{t} \xi(x, t)+\Lambda_{\sigma\left(t_{1}\right)} \partial_{x} \xi(x, t)=0,
$$

for all $x \in(0,1)$, with the corresponding boundary condition

$$
\left[\begin{array}{l}
\xi_{-}^{\sigma\left(t_{1}\right)}(1, t) \\
\xi_{+}^{\sigma\left(t_{1}\right)}(0, t)
\end{array}\right]=G_{\sigma\left(t_{1}\right)}\left[\begin{array}{l}
\xi_{-}^{\sigma\left(t_{1}\right)}(0, t) \\
\xi_{+}^{\sigma\left(t_{1}\right)}(1, t)
\end{array}\right],
$$

and the redefined initial condition $\xi^{1} \in L^{2}(0,1)$. Again applying (Bastin and Coron, 2016, Theorem A.4), for the initial-boundary value problem (8)-(9) of the mode $\sigma\left(t_{1}\right) \in \mathcal{S}$, as $t \in\left[t_{1} \wedge T, t_{2} \wedge T\right]$, there also exists a unique classical solution $\xi(\cdot, t) \in L^{2}(0,1)$, which satisfies

$$
\|\xi(\cdot, t)\|_{L^{2}(0,1)} \leq C_{2}\left\|\xi^{1}(\cdot)\right\|_{L^{2}(0,1)},
$$

with $C_{2}>0$, then it holds $E\left\{\left\|\xi\left(\cdot, t_{2} \wedge T\right)\right\|_{L^{2}(0,1)}\right\}<$ $\infty$. Repeating above procedure, we see that the MJLH system (2)-(3) has a unique solution $\xi(\cdot, t)$ for any $t \in$ $[0, T]$, and satisfies $E\left\{\|\xi(\cdot, T)\|_{L^{2}(0,1)}\right\}<\infty$. Since time $T$ is arbitrary, this concludes the proof of Proposition 1.

\section{Stochastic Stability of Markov Jump Linear Hyperbolic Systems}

Stochastic stability is an important issue in the analysis of stochastic systems. For the MJL systems, various definitions have been introduced in Costa et al. (2013), and Fang and Loparo (2002).

We next start this section by defining the exponential mean-square stability for MJLH systems.

Definition 1 System (2)-(4) is said to be exponentially mean-square stable, if there exist $\nu>0$ and $C>0$, such that any solution $\xi(\cdot, t)$ to (2)-(4) satisfies

$$
E\left\{\|\xi(\cdot, t)\|_{L^{2}(0,1)}^{2}\right\} \leq C e^{-\nu t}\left\|\xi^{0}(\cdot)\right\|_{L^{2}(0,1)}^{2},
$$

for all $t \in \mathbb{R}_{+}$, any initial condition $\xi^{0}(\cdot) \in L^{2}(0,1)$ and any initial operation mode $\sigma(0) \in \mathcal{S}$.

A sufficient condition for the exponential mean-square stability of MJLH systems is obtained using the Lyapunov function method. To do that, let us consider the following candidate stochastic Lyapunov function for the MJLH system (2)-(4)

$$
V(\xi, \sigma(t))=\int_{0}^{1} \xi(x, t)^{\top} P(x, \sigma(t)) \xi(x, t) d x,
$$


with $P(x, \sigma(t)=i)=P_{i}(x), i \in \mathcal{S}$. We define $P_{i}(x)=$ $\operatorname{diag}\left\{e^{2 \mu_{i} x} P_{i}^{-}, e^{-2 \mu_{i} x} P_{i}^{+}\right\}, x \in[0,1]$, where $\mu_{i} \in \mathbb{R}$, $P_{i}^{-} \in \mathcal{D}_{+}^{m_{i} \times m_{i}}$ and $P_{i}^{+} \in \mathcal{D}_{+}^{\left(n-m_{i}\right) \times\left(n-m_{i}\right)}$ are diagonal positive definite matrices with corresponding dimensions.

Definition 2 The infinitesimal generator of the solution process $(\xi(\cdot, t), \sigma(t))$ of the MJLH system (2)-(4), acting on the Lyapunov function $V(\xi, \sigma(t))$ at the point $(t, \xi(\cdot, t), \sigma(t)=i), i \in \mathcal{S}$, is defined by, for all $t \in \mathbb{R}_{+}$,

$$
\begin{aligned}
& \mathcal{L} V(\xi(\cdot, t), \sigma(t)=i) \\
& \triangleq \lim _{\Delta t \rightarrow 0^{+}} \frac{1}{\Delta t}[E\{V(\xi(\cdot, t+\Delta t), \sigma(t+\Delta t)) \mid \\
& \quad \xi(\cdot, t), \sigma(t)=i\}-V(\xi(\cdot, t), \sigma(t)=i)] .
\end{aligned}
$$

As the point $t$ is clear in the context, we denote $V(\xi, i)=$ $V(\xi, \sigma(t)=i)$ for simplicity.

Lemma 1 Given the stochastic Lyapunov function (12) and the MJLH system (2)-(4), the infinitesimal generator of Lyapunov function defined in (13), satisfies

$$
\mathcal{L} V(\xi, \sigma(t)=i)=\frac{d}{d t} V(\xi, i)+\sum_{j=1}^{N} \pi_{i j} V(\xi, j),
$$

where derivative $\frac{d}{d t} V(\xi, i)$ stands for $\frac{\partial}{\partial \xi} V(\xi, i) \partial_{t} \xi$ at the point $(t, \xi(\cdot, t), \sigma(t)=i)$ for any solution to (2)-(4).

Proof. The weighting matrix $P(x, \sigma(t))$ of the Lyapunov function (12) is also a Markov process and is equipped with the transition probabilities

$$
\begin{aligned}
& \operatorname{Pr}\left\{P\left(\cdot, \sigma(t+\Delta t)=P_{j}(\cdot) \mid \xi(\cdot, t), \sigma(t)=i\right\}\right. \\
& =\pi_{i j} \Delta t+o(\Delta t), \quad \text { if } i \neq j
\end{aligned}
$$

It follows from (1) and (2) that

$$
\begin{aligned}
\operatorname{Pr}\{\xi(\cdot, t+\Delta t)= & \xi(\cdot, t)-\Lambda_{j} \partial_{x} \xi(\cdot, t) \Delta t+o(\Delta t) \\
& \mid \xi(\cdot, t), \sigma(t)=i\} \\
= & \pi_{i j} \Delta t+o(\Delta t), \text { if } i \neq j \\
& \\
\operatorname{Pr}\{\xi(\cdot, t+\Delta t)= & \xi(\cdot, t)-\Lambda_{i} \partial_{x} \xi(\cdot, t) \Delta t+o(\Delta t) \\
& \mid \xi(\cdot, t), \sigma(t)=i\} \\
= & 1+\pi_{i i} \Delta t+o(\Delta t), \text { if } i=j
\end{aligned}
$$

Using the properties of the conditional expectation, we have

$$
\begin{aligned}
& E\{V(\xi(\cdot, t+\Delta t), \sigma(t+\Delta t)) \mid \xi(\cdot, t), \sigma(t)=i\} \\
=[1+ & \left.\pi_{i i} \Delta t\right] V(\xi(\cdot, t+\Delta t), i) \\
& +\sum_{j=1, j \neq i}^{N}\left[\pi_{i j} \Delta t\right] V(\xi(\cdot, t+\Delta t), j)+o(\Delta t) \\
=V(\xi(\cdot, t+\Delta t), i) & \\
& +\sum_{j=1}^{N}\left[\pi_{i j} \Delta t\right] V(\xi(\cdot, t+\Delta t), j)+o(\Delta t) .
\end{aligned}
$$

Hence, in mode $i \in \mathcal{S}$, when the system state is changing from $\xi(\cdot, t)$ to $\xi(\cdot, t+\Delta t)$, we have

$$
\begin{aligned}
& V(\xi(\cdot, t+\Delta t), i) \\
= & \int_{0}^{1} \xi^{\top}(\cdot, t+\Delta t) P_{i}(\cdot) \xi(\cdot, t+\Delta t) d x \\
= & V(\xi(\cdot, t), i)-2 \Delta t \int_{0}^{1} \xi^{\top}(\cdot, t) \Lambda_{i} P_{i}(\cdot) \partial_{x} \xi(\cdot, t) d x \\
& \quad+o(\Delta t) \\
= & V(\xi(\cdot, t), i)+\Delta t \int_{0}^{1}\left[\partial_{t} \xi^{\top}(\cdot, t) P_{i}(\cdot) \xi(\cdot, t)\right. \\
& \left.\quad+\xi^{\top}(\cdot, t) P_{i}(\cdot) \partial_{t} \xi(\cdot, t)\right] d x+o(\Delta t) \\
= & V(\xi(\cdot, t), i)+\Delta t \frac{d}{d t} V(\xi(\cdot, t), i)+o(\Delta t) .
\end{aligned}
$$

From the definition of function $\mathcal{L} V$ (13), it follows that

$$
\begin{aligned}
& \mathcal{L} V(\xi(\cdot, t), \sigma(t)=i) \\
= & \lim _{\Delta t \rightarrow 0^{+}} \frac{1}{\Delta t}\left\{V(\xi(\cdot, t+\Delta t), i)+\sum_{j=1}^{N}\left[\pi_{i j} \Delta t\right]\right. \\
& {[V(\xi(\cdot, t+\Delta t), j)-V(\xi(\cdot, t), i)]+o(\Delta t)\} } \\
= & \lim _{\Delta t \rightarrow 0^{+}} \frac{1}{\Delta t}[V(\xi(\cdot, t+\Delta t), i)-V(\xi(\cdot, t), i)] \\
& +\sum_{j=1}^{N} \pi_{i j} V(\xi, j) \\
= & \frac{d}{d t} V(\xi, i)+\sum_{j=1}^{N} \pi_{i j} V(\xi, j) .
\end{aligned}
$$

This concludes the proof of Lemma 1.

The following Theorem 1 captures the idea that seeking a balance, between the boundary conditions of hyperbolic modes and the transition probability of the Markov process, is essential for the exponential mean-square stability of MJLH systems. Let $\left|\Lambda_{i}\right|=\operatorname{diag}\left\{\left|\lambda_{1}^{i}\right|, \cdots,\left|\lambda_{n}^{i}\right|\right\}$, $i \in \mathcal{S}$, and the matrices

$$
G_{i-}=\left[\begin{array}{cc}
G_{--}^{i} & G_{-+}^{i} \\
0 & I_{n-m_{i}}
\end{array}\right], G_{i+}=\left[\begin{array}{cc}
I_{m_{i}} & 0 \\
G_{+-}^{i} & G_{++}^{i}
\end{array}\right] .
$$


Theorem 1 Let us assume that there exist $\nu>0, \mu_{i} \in$ $\mathbb{R}, i \in \mathcal{S}$, and diagonal positive definite matrices $P_{i}^{-} \in$ $\mathcal{D}_{+}^{m_{i} \times m_{i}}$ and $P_{i}^{+} \in \mathcal{D}_{+}^{\left(n-m_{i}\right) \times\left(n-m_{i}\right)}$ such that $P_{i}(x)=$ $\operatorname{diag}\left\{e^{2 \mu_{i} x} P_{i}^{-}, e^{-2 \mu_{i} x} P_{i}^{+}\right\}$, defined for each $x \in[0,1]$, satisfies the following matrix inequalities:

$$
\begin{gathered}
-2 \mu_{i}\left|\Lambda_{i}\right| P_{i}(x)+\sum_{j=1}^{N} \pi_{i j} P_{j}(x) \leq-\nu P_{i}(x), \\
G_{i+}^{\top} \Lambda_{i} P_{i}(0) G_{i+}-G_{i-}^{\top} \Lambda_{i} P_{i}(1) G_{i-} \leq 0 .
\end{gathered}
$$

Then, there exists $C>0$ such that (11) holds and the MJLH system (2)-(4) is exponentially mean-square stable.

Proof. Let us consider the Lyapunov function candidate $V(\xi, \sigma(t))$ given by (12) and assume that the diagonal positive matrix $P_{i}(x)$ satisfies the inequalities $(21)$ and $(22)$, for each $i \in \mathcal{S}$. Given any $t \in \mathbb{R}_{+}$with the Markov process $\sigma(t)=i$, computing the time-derivative of $V(\xi, i)$ along the solutions to (2) yields the following: $\frac{d}{d t} V(\xi, i)=\int_{0}^{1}\left[\xi^{\top} P_{i}(x) \partial_{t} \xi+\partial_{t} \xi^{\top} P_{i}(x) \xi\right] d x=$ $-\int_{0}^{1}\left[\xi^{\top} P_{i}(x) \Lambda_{i} \partial_{x} \xi+\partial_{x} \xi^{\top} \Lambda_{i} P_{i}(x) \xi\right] d x$. Using an integration by parts, and because $\partial_{x} P_{i}(x) \Lambda_{i}=$ $-2 \mu_{i} P_{i}(x)\left|\Lambda_{i}\right|$, we obtain

$$
\begin{aligned}
& \frac{d}{d t} V(\xi, i) \\
= & -\left[\xi^{\top} P_{i}(x) \Lambda_{i} \xi\right]_{0}^{1}-\int_{0}^{1} 2 \mu_{i} \xi^{\top}\left|\Lambda_{i}\right| P_{i}(x) \xi d x \\
= & \xi^{\top}(0, t) P_{i}(0) \Lambda_{i} \xi(0, t)-\xi^{\top}(1, t) P_{i}(1) \Lambda_{i} \xi(1, t) \\
& -2 \int_{0}^{1} \xi^{\top} \mu_{i}\left|\Lambda_{i}\right| P_{i}(x) \xi d x \\
= & \partial_{t} V_{1}(\xi, i)+\partial_{t} V_{2}(\xi, i),
\end{aligned}
$$

with

$$
\begin{gathered}
\partial_{t} V_{1}(\xi, i) \triangleq \xi^{\top}(0, t) P_{i}(0) \Lambda_{i} \xi(0, t) \\
-\xi^{\top}(1, t) P_{i}(1) \Lambda_{i} \xi(1, t), \\
\partial_{t} V_{2}(\xi, i) \triangleq-2 \int_{0}^{1} \xi^{\top} \mu_{i}\left|\Lambda_{i}\right| P_{i}(x) \xi d x .
\end{gathered}
$$

Under the boundary condition (3) for mode $i$, we have

$$
\begin{aligned}
\partial_{t} V_{1}(\xi, i)= & {\left[\begin{array}{c}
\xi_{-}^{i}(0, t) \\
\xi_{+}^{i}(1, t)
\end{array}\right]^{\top}\left(\left[\begin{array}{cc}
I_{m_{i}} & 0 \\
G_{+-}^{i} & G_{++}^{i}
\end{array}\right]^{\top} \Lambda_{i} P_{i}(0)\right.} \\
& {\left[\begin{array}{cc}
I_{m_{i}} & 0 \\
G_{+-}^{i} & G_{++}^{i}
\end{array}\right]-\left[\begin{array}{cc}
G_{--}^{i} & G_{-+}^{i} \\
0 & I_{n-m_{i}}
\end{array}\right]^{\top} \Lambda_{i} P_{i}(1) } \\
& {\left.\left[\begin{array}{cc}
G_{--}^{i} & G_{-+}^{i} \\
0 & I_{n-m_{i}}
\end{array}\right]\right)\left[\begin{array}{c}
\xi_{-}^{i}(0, t) \\
\xi_{+}^{i}(1, t)
\end{array}\right] }
\end{aligned}
$$

$$
\begin{gathered}
=\left[\begin{array}{c}
\xi_{-}^{i}(0, t) \\
\xi_{+}^{i}(1, t)
\end{array}\right]^{\top}\left(G_{i+}^{\top} \Lambda_{i} P_{i}(0) G_{i+}\right. \\
\left.-G_{i-}^{\top} \Lambda_{i} P_{i}(1) G_{i-}\right)\left[\begin{array}{c}
\xi_{-}^{i}(0, t) \\
\xi_{+}^{i}(1, t)
\end{array}\right] .
\end{gathered}
$$

Combining the time-derivatives (23), (24) and inequality conditions (21), (22) and Lemma 1, the infinitesimal generator of $V(\xi, \sigma(t))$ satisfies

$$
\begin{aligned}
\mathcal{L} V & (\xi, \sigma(t)=i)=\frac{d}{d t} V(\xi, i)+\sum_{j=1}^{N} \pi_{i j} V(\xi, j) \\
& =\partial_{t} V_{1}(\xi, i)+\partial_{t} V_{2}(\xi, i)+\sum_{j=1}^{N} \pi_{i j} V(\xi, j) \\
& \leq \int_{0}^{1} \xi^{\top}\left[-2 \mu_{i}\left|\Lambda_{i}\right| P_{i}(x)+\sum_{j=1}^{N} \pi_{i j} P_{j}(x)\right] \xi d x . \\
& \leq-\nu V(\xi, i) .
\end{aligned}
$$

Now, we can define a new Lyapunov function $\tilde{V}(\xi, \sigma(t))$ as $\tilde{V}(\xi, \sigma(t)=i)=e^{\nu t} V(\xi, \sigma(t)=i)$, for all $i \in \mathcal{S}$. From Lemma 1 and (25), we have

$$
\mathcal{L} \tilde{V}(\xi, \sigma(t)=i)=\mathcal{L} V(\xi, \sigma(t)=i)+\nu V(\xi, i) \leq 0
$$

Based on the definition of $\mathcal{L} \tilde{V}$, for each $\sigma(t)=i \in \mathcal{S}$, $t \geq 0$, integrating (26) from zero to $t$, we get

$$
\begin{aligned}
E\left\{e^{\nu t} V(\xi, \sigma(t))\right\} & -V\left(\xi^{0}(x), \sigma(0)\right) \\
& =E\left\{\int_{0}^{t} \mathcal{L} \tilde{V}(\xi, \sigma(\tau)) d \tau\right\} \leq 0
\end{aligned}
$$

Therefore $E\left\{V(\xi(x, t), \sigma(t)) \mid \xi^{0}, \sigma(0)\right\} \leq V\left(\xi^{0}, \sigma(0)\right) e^{-\nu t}$ for any initial condition $\xi^{0} \in L^{2}(0,1)$ and any initial operation mode $\sigma(0) \in \mathcal{S}$. By remarking that there exist $\alpha>0, \beta>0$ (depending on $\mu_{i}, P_{i}^{+}$and $P_{i}^{-}$, for all $i \in \mathcal{S})$, such that

$$
\alpha\|\xi(x, t)\|_{L^{2}(0,1)}^{2} \leq V(\xi(x, t), \sigma(t)) \leq \beta\|\xi(x, t)\|_{\left.L^{2}(0,1)\right)}^{2},
$$

we obtain that, for all $t \in \mathbb{R}_{+}$,

$$
\begin{aligned}
\alpha E\left\{\|\xi(t, x)\|_{L^{2}(0,1)}^{2}\right\} & \leq E\{V(\xi(t, x), \sigma(t))\} \\
& \leq \beta\left\|\xi^{0}(x)\right\|_{L^{2}(0,1)}^{2} e^{-\nu t}
\end{aligned}
$$

which straightly implies

$$
E\left\{\|\xi(t, x)\|_{L^{2}(0,1)}^{2}\right\} \leq \alpha^{-1} \beta e^{-\nu t}\left\|\xi^{0}(x)\right\|_{L^{2}(0,1)}^{2} .
$$

Consequently, the MJLH system (2)-(4) is exponentially mean-square stable in $L^{2}$-norm by choosing $C=\alpha^{-1} \beta$. This completes the proof of Theorem 1. 
Remark 1 For the case of only one mode of operation in the MJLH system (2)-(4), $N=1$, that is no jumps in the Markov process, condition (21) in Theorem 1 implies the parameter $\mu_{1}>0$, and furthermore condition (22) in Theorem 1 is consistent with the dissipative boundary conditions for the linear hyperbolic systems of conservation laws by Coron et al. (2007).

The condition (21) of Theorem 1 involves the spatial variable, then the number of inequality constraints is infinite. The following Corollary 1 presents a more easily checked matrix inequality condition by testing the upper bound of the involved spatial variable $x \in[0,1]$. To do that, denote $P_{i}=\operatorname{diag}\left\{P_{i}^{-}, P_{i}^{+}\right\}$and

$$
I_{i}(x)=\operatorname{diag}\left\{e^{2 \mu_{i} x} I_{m_{i}}, e^{-2 \mu_{i} x} I_{n-m_{i}}\right\},
$$

which implies $P_{i}(x)=P_{i} I_{i}(x)$, for all $x \in[0,1]$.

Corollary 1 Let us assume there exist $\mu_{i} \in \mathbb{R}$ and diagonal positive definite matrices $P_{i} \in \mathcal{D}_{+}^{n \times n}$ such that the following matrix inequalities hold, for all $i, j \in \mathcal{S}$

$$
\begin{array}{r}
\left(\pi_{i i} I_{n}-2 \mu_{i}\left|\Lambda_{i}\right|\right) P_{i}+e^{2\left|\mu_{i}\right|} \sum_{j \neq i}^{N} \pi_{i j} e^{2\left|\mu_{j}\right|} P_{j}<0, \\
G_{i+}^{\top} \Lambda_{i} P_{i} G_{i+}-G_{i-}^{\top} \Lambda_{i} P_{i} I_{i}(1) G_{i-} \leq 0 .
\end{array}
$$

Then the MJLH system (2)-(4) is exponentially meansquare stable.

Proof. The matrix inequality (30) implies that there exists a common positive small number $\nu>0$ (depends on matrices $P_{i},\left|\Lambda_{i}\right|$, and $\left.\mu_{i}, \pi_{i j}, i, j \in \mathcal{S}\right)$, such that

$$
\left(\pi_{i i} I_{n}-2 \mu_{i}\left|\Lambda_{i}\right|\right) P_{i}+e^{2\left|\mu_{i}\right|} \sum_{j \neq i}^{N} \pi_{i j} e^{2\left|\mu_{j}\right|} P_{j} \leq-\nu P_{i} .
$$

Multiplying $I_{i}(x)$ on both sides of (32), we obtain $-2 \mu_{i}\left|\Lambda_{i}\right| P_{i}(x)+\pi_{i i} P_{i}(x)+e^{2\left|\mu_{i}\right|} \sum_{j \neq i}^{N} \pi_{i j} e^{2\left|\mu_{j}\right|} P_{j} I_{i}(x) \leq$ $-\nu P_{i}(x)$. Since $\pi_{i j} \geq 0$, and $e^{2\left|\mu_{i}\right|} I_{i}(x) \geq 1$, we have $\sum_{j=1, j \neq i}^{N} \pi_{i j} P_{j}(x) \leq e^{2\left|\mu_{i}\right|} \sum_{j \neq i}^{N} \pi_{i j} e^{2\left|\mu_{j}\right|} P_{j} I_{i}(x)$, thus the condition (21) of Theorem 1 holds. This concludes the proof of Corollary 1.

Compared with the results of Theorem 1, the sufficient conditions (30)-(31) of Corollary 1 remain nonlinear in the unknown variables $\mu_{i}$ and $P_{i}, i \in \mathcal{S}$. However, since parameter $\mu_{i}$ is a scalar variable, one may use a line search algorithm to solve inequalities (30) and (31) (see Section 4 below).

\section{Numerical Computation for Stochastic Sta- bility Conditions}

In this section, a bounded real interval of the parameter $\mu_{i}$ is obtained by a trade-off between the condition (21) and (22) of Theorem 1. We define, for each $i \in \mathcal{S}$,

$$
\begin{aligned}
& \mu_{i}^{\pi}=\lambda_{\max }\left(\frac{\pi_{i i}}{2}\left|\Lambda_{i}\right|^{-1}\right), \\
& \mu_{i}^{G}=-\ln \rho\left(G_{i}\right) .
\end{aligned}
$$

Proposition 2 For a given mode $i \in \mathcal{S}$, conditions (21) and (22) of Theorem 1 are both satisfied, only if $\mu_{i}^{\pi}<$ $\mu_{i} \leq \mu_{i}^{G}$.

Proof. For a given parameter $\mu_{i} \in \mathbb{R}$, we first prove that $\mu_{i}>\mu_{i}^{\pi}$. On the one hand, for all $x \in[0,1]$, condition (21) may be developed as $\left(-\mu_{i}\left|\Lambda_{i}\right|\right)^{\top} P_{i}(x)+$ $P_{i}(x)\left(-\mu_{i}\left|\Lambda_{i}\right|\right)+\sum_{j=1}^{N} \pi_{i j} P_{j}(x) \leq-\nu P_{i}(x)$. By letting $x=0$, condition (21) includes a finite number of constraints and describes the stochastic stability of the following continuous-time MJL system

$$
\dot{y}(t)=-\mu_{i}\left|\Lambda_{i}\right| y(t) .
$$

Let $A_{i}=-\mu_{i}\left|\Lambda_{i}\right|, \otimes$ denotes the Kronecker product and $\oplus$ denotes the Kronecker sum, i.e., $A \oplus B=A \otimes I+$ $B \otimes I$, and just recalling the well-known result for the exponentially mean-square stable of MJL (see e.g. Fang and Loparo (2002)), the $n^{2} N \times n^{2} N$ matrix $\Gamma$ defined in (44) is Hurwitz.

Note that $\pi_{i j}>0$ for all $i \neq j, i, j \in \mathcal{S}$, then the matrix $A_{i}+\frac{\pi_{i i}}{2} I_{n}$ is Hurwitz which requires the largest real part of all eigenvalues being non-positive. Hence, requiring the parameter $\mu_{i}>\lambda_{\max }\left(\frac{\pi_{i i}}{2}\left|\Lambda_{i}\right|^{-1}\right)$ is necessary to satisfy the condition (21) of Theorem 1 .

On the other hand, condition (22) of Theorem 1 may be described as

$$
Q_{i}=\left[\begin{array}{ll}
Q_{--}^{i} & Q_{-+}^{i} \\
Q_{+-}^{i} & Q_{++}^{i}
\end{array}\right] \leq 0,
$$

with $Q_{--}^{i}=\left(G_{+-}^{i}\right)^{\top} \Lambda_{i}^{+} P_{i}^{+} G_{+-}^{i}+e^{2 \mu_{i}}\left(G_{--}^{i}\right)^{\top}\left|\Lambda_{i}^{-}\right| P_{i}^{-} G_{--}^{i}-$ $\left|\Lambda_{i}^{-}\right| P_{i}^{-}, Q_{+-}^{i}=\left(G_{+-}^{i}\right)^{\top} \Lambda_{i}^{+} P_{i}^{+} G_{++}^{i}+e^{2 \mu_{i}}\left(G_{--}^{i}\right)^{\top}\left|\Lambda_{i}^{-}\right| P_{i}^{-} G_{-+}^{i}$, $Q_{-+}^{i}=\left(Q_{+-}^{i}\right)^{\top}, Q_{++}^{i}=\left(G_{++}^{i}\right)^{\top} \Lambda_{i}^{+} P_{i}^{+} G_{++}^{i}+$ $e^{2 \mu_{i}}\left(G_{-+}^{i}\right)^{\top}\left|\Lambda_{i}^{-}\right| P_{i}^{-} G_{-+}^{i}-e^{-2 \mu_{i}} \Lambda_{i}^{+} P_{i}^{+}$, where $\left|\Lambda_{i}^{-}\right|=$ $\operatorname{diag}\left\{\left|\lambda_{1}^{i}\right|, \cdots,\left|\lambda_{m_{i}}^{i}\right|\right\}$, and $\Lambda_{i}^{+}=\operatorname{diag}\left\{\lambda_{m_{i}+1}^{i}, \cdots, \lambda_{n}^{i}\right\}$.

Then the matrix $Q_{i}$ in (45) can be rewritten as

$$
\begin{gathered}
Q_{i}=\left(e_{i}^{\mu} G_{i}\right)^{\top} \operatorname{diag}\left\{\left|\Lambda_{i}^{-}\right| P_{i}^{-}, e^{-2 \mu_{i}} \Lambda_{i}^{+} P_{i}^{+}\right\} e_{i}^{\mu} G_{i} \\
-\operatorname{diag}\left\{\left|\Lambda_{i}^{-}\right| P_{i}^{-}, e^{-2 \mu_{i}} \Lambda_{i}^{+} P_{i}^{+}\right\} .
\end{gathered}
$$

It implies that the discrete-time system

$$
y(k+1)=e^{\mu_{i}} G_{i} y(k), \quad k=0,1, \cdots
$$

is marginally stable.

Hence, for the system matrix $e^{\mu_{i}} G_{i}$ of (47), we have $e^{\mu_{i}} \rho\left(G_{i}\right) \leq 1$. Thus, the inequality $\mu_{i} \leq-\ln \rho\left(G_{i}\right)$ is necessary to satisfy the condition (22) of Theorem 1 . This concludes the proof of Proposition 2. 


$$
\Gamma=\left[\begin{array}{cccc}
A_{1} \oplus A_{1}+\pi_{11} I_{n^{2}} & \pi_{21} I_{n^{2}} & \cdots & \pi_{N 1} I_{n^{2}} \\
\pi_{12} I_{n^{2}} & A_{2} \oplus A_{2}+\pi_{22} I_{n^{2}} & \cdots & \pi_{N 2} I_{n^{2}} \\
\vdots & \vdots & \ddots & \vdots \\
\pi_{1 N} I_{n^{2}} & \pi_{2 N} I_{n^{2}} & \cdots & A_{N} \oplus A_{N}+\pi_{N N} I_{n^{2}}
\end{array}\right]
$$

Remark 2 Following the proof of Corollary 1, if the conditions (30) and (31) of Corollary 1 are satisfied, then, by Proposition 2 , it holds $\mu_{i} \in\left(\mu_{i}^{\pi}, \mu_{i}^{G}\right]$. Therefore, when using a branch and bound algorithm for finding the parameters $\mu_{i}, i \in \mathcal{S}$, one can further limit the search range of $\mu_{i}$ among the initial interval $\left(\mu_{i}^{\pi}, \mu_{i}^{G}\right]$.

\section{Application to Boundary Control of Freeway Traffic}

\subsection{Aw-Rascle traffic flow model}

The traffic dynamic in a freeway section of length $L$ is governed by a quasi-linear hyperbolic system including two equations, the so-called Aw-Rascle traffic flow model (Aw and Rascle (2000)). That is

$$
\left\{\begin{array}{l}
\partial_{t} \rho+\partial_{x}(\rho v)=0 \\
\partial_{t}(v+p(\rho))+v \partial_{x}(v+p(\rho))=0
\end{array}\right.
$$

where $\rho(x, t)$ is vehicle density, $v(x, t)$ is average speed, $x \in[0, L], t \geq 0$, and the function $p(\rho)$ means the traffic pressure which is supposed to be increasing against density. In Zhang (2002), pressure function $p(\rho)$ is given as

$$
p(\rho)=v_{f}-V(\rho),
$$

where $v_{f}$ is the free (maximal) speed. Typically, from the Greenshields fundamental diagram (Greenshields (1935)), define

$$
V(\rho)=v_{f}\left(1-\frac{\rho}{\rho_{m}}\right),
$$

where $\rho_{m}$ is the maximal density. Thus, we get a linear pressure function $p(\rho)=a \rho$ with $a=\frac{v_{f}}{\rho_{m}}$.

Let $z=v$, and $w=v+a \rho$, the Aw-Rascle equation (48) may be rewritten in the characteristic Riemann coordinates as

$$
\partial_{t} \xi(x, t)+\Lambda(\xi) \partial_{x} \xi(x, t)=0,
$$

with the system matrix $\Lambda(\xi)=\operatorname{diag}\{2 z-w, z\}$ and the state $\xi=[z, w]^{\top}$. Equation (51) is assumed to be strictly hyperbolic as the characteristic velocities $\lambda_{1}=2 z-w=$ $v-a \rho, \lambda_{2}=z=v$ are different and nonzero for all $x \in[0, L], t \geq 0$.
In (51), the sign of the first characteristic velocity $\lambda_{1}>$ 0 , (or $<0$ ), indicates the transfer direction of traffic information, such as the average speed $z$ (or $v$ ), from the freeway upstream to the downstream, or inverse. It is natural as the feature to determine the freeway traffic lies in the free-flow traffic mode or in the congestion traffic mode (Kerner (2009) $)$.

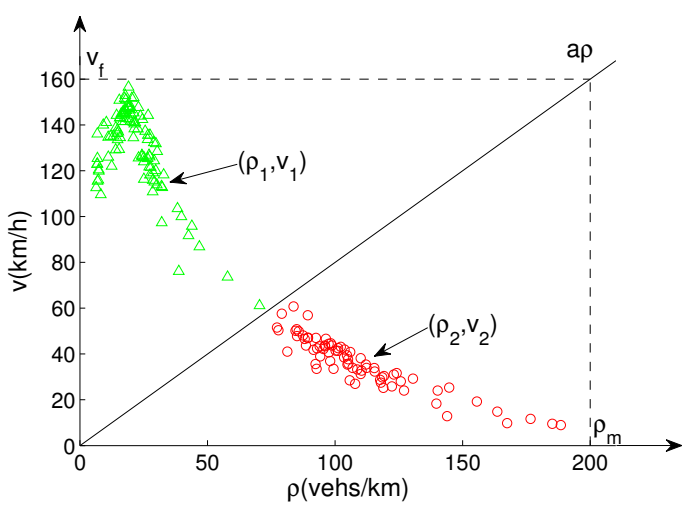

Figure 1. Relation of the speed and density. The experiment data have been recorded on I80 freeway in Emeryville, CA, 4:00-4:30 pm, June 1, 2015, by PeMS (2004).

\subsection{MJLH traffic flow model and linearization}

Motivated by the development of stochastic traffic flow models, see Boel and Mihaylova (2006), Zhang and Mao (2015), we deduce the quasi-linear hyperbolic equation (48) to a MJLH system.

Firstly, we choose two typical traffic states $\xi_{1}=\left(\rho_{1}, v_{1}\right)$ and $\xi_{2}=\left(\rho_{2}, v_{2}\right)$ from the separated regions of the fundamental diagram, with $v_{1}-a \rho_{1}>0$ and $v_{2}-a \rho_{2}<0$, respectively, to represent the characteristics of the freeflow and the congestion modes, as shown in Fig. 1.

In Riemann coordinates, the system matrices of the MJLH system (2) for free-flow or congestion modes are given respectively by $\Lambda_{1}=\Lambda\left(\xi_{1}\right)=\operatorname{diag}\left\{2 z_{1}-w_{1}, z_{1}\right\}$, $\Lambda_{2}=\Lambda\left(\xi_{2}\right)=\operatorname{diag}\left\{2 z_{2}-w_{1}, z_{2}\right\}$. We further assume the probabilistic conditions of mode switching follows a Markov process $\sigma(t)$ under a prior determined infinitesimal generator $\Pi \in \mathbb{R}^{2 \times 2}$, see Sumalee et al. (2011).

Hence, the MJLH traffic flow model becomes

$$
\partial_{t} \xi(x, t)+\Lambda_{\sigma(t)} \partial_{x} \xi(x, t)=0,
$$


with $\sigma(t) \in\{1,2\}$, in which $\sigma(t)=1$ means the freeway traffic lies in the free-flow mode, and $\sigma(t)=2$ means the congestion mode.

A steady-state of freeway traffic is a constant traffic state $\left(\rho^{*}, v^{*}\right)$ which satisfies the flux conservation condition at the left boundary

$$
p_{i n}+r_{0}=\rho^{*} v^{*},
$$

where $p_{i n}$ and $r_{0}$ are constant flux of driving-in vehicles through the mainline and the on-ramp of the freeway section, respectively. Then, the associated steady-state $\xi^{*}=\left(z^{*}, w^{*}\right)^{\top}$ in Riemann coordinates is given as $z^{*}=$ $v^{*}$ and $w^{*}=v^{*}+a \rho^{*}$. We define the deviations of traffic state $(\rho(x, t), v(x, t))$ with respect to the steady-state $\left(\rho^{*}, v^{*}\right)$ as $\widetilde{\rho}=\rho-\rho^{*}$, and $\widetilde{v}=v-v^{*}$.The MJLH system (52) around the steady-state $\xi^{*}$ is derived as

$$
\partial_{t} \widetilde{\xi}(x, t)+\Lambda_{\sigma(t)} \partial_{x} \widetilde{\xi}(x, t)=0,
$$

where the deviation $\widetilde{\xi}=\xi-\xi^{*}$ includes two entries $\widetilde{z}=$ $z-z^{*}$ and $\widetilde{w}=w-w^{*}$.

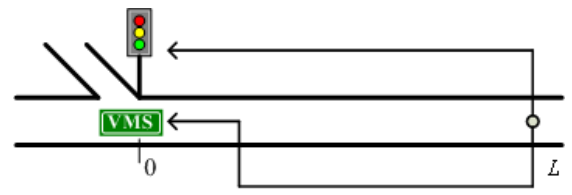

Figure 2. On-ramp metering and variable speed limit on a freeway section.

\subsection{Boundary feedback control}

We now are going to show how Corollary 1 may be applied to analyze the stochastic stability of the MJLH model (52). The boundary control applies the proportional feedback by combining the on-ramp metering $u(t)$ and the speed limit $v(0, t)$, which are all stored at the upstream boundary. Traffic measurements are density $\rho(L, t)$ and average speed $v(L, t)$ at the downstream boundary, see Fig. 2.

Precisely, we introduce the boundary feedback control:

$$
\left\{\begin{array}{l}
u(t)=r_{0}-k_{\rho}\left(\rho(L, t)-\rho^{*}\right) \\
v(0, t)=v^{*}-k_{v}\left(v(L, t)-v^{*}\right)
\end{array},\right.
$$

where $k_{\rho} \in \mathbb{R}$ and $k_{v} \in \mathbb{R}$ are proportional gains to be designed. In order to get the boundary conditions corresponding to the Riemann invariant (54), two cases are discussed separately.

Case 1: Free-flow mode for the system matrix $\Lambda_{1}$.

At the left boundary of the freeway section, i.e., $x=0$, the driving-in flux conservation equation holds

$$
u(t)+p_{\text {in }}=\rho(0, t) v(0, t) .
$$

After the linearization of boundary condition (56) with integrating the feedback control law (55), we have the following boundary condition

$$
\widetilde{\rho}(0, t)=\rho^{*} \frac{k_{v}}{v^{*}} \widetilde{v}(L, t)-\frac{k_{\rho}}{v^{*}} \widetilde{\rho}(L, t) .
$$

Rewrite condition (57) in Riemann coordinates as

$$
\begin{aligned}
\widetilde{w}(0, t)= & -\frac{k_{\rho}}{v^{*}} \widetilde{w}(L, t) \\
& +\left[a \rho^{*} \frac{k_{v}}{v^{*}}+\frac{k_{\rho}}{v^{*}}-k_{v}\right] \widetilde{z}(L, t) .
\end{aligned}
$$

Since $\widetilde{v}(0, t)=-k_{v} \widetilde{v}(L, t)$, and $\widetilde{v}=\widetilde{z}$, the boundary condition that needs to be imposed for the free flow mode can be written as

$$
\left[\begin{array}{c}
\widetilde{z}(0, t) \\
\widetilde{w}(0, t)
\end{array}\right]=G_{1}\left[\begin{array}{l}
\widetilde{z}(L, t) \\
\widetilde{w}(L, t)
\end{array}\right],
$$

with $G_{1}=\left[\begin{array}{cc}-k_{v} & 0 \\ a \rho^{*} \frac{k_{v}}{v^{*}}+\frac{k_{\rho}}{v^{*}}-k_{v} & -\frac{k_{\rho}}{v^{*}}\end{array}\right]$.

Case 2: Congestion mode for the system matrix $\Lambda_{2}$.

In this case, using the same feedback control (55), we have $\widetilde{v}(L, t)=-\frac{1}{k_{v}} \widetilde{v}(0, t)$. Similarly, in the Riemann coordinates, it holds

$$
\begin{aligned}
\widetilde{w}(0, t)= & -\frac{k_{\rho}}{v^{*}} \widetilde{w}(L, t) \\
& +\left[1-\frac{a \rho^{*}}{v^{*}}-\frac{k_{\rho}}{v^{*} k_{v}}\right] \widetilde{z}(0, t) .
\end{aligned}
$$

Hence, the boundary condition that needs to be imposed for the congestion mode is written as

$$
\begin{gathered}
\qquad\left[\begin{array}{c}
\widetilde{z}(L, t) \\
\widetilde{w}(0, t)
\end{array}\right]=G_{2}\left[\begin{array}{c}
\widetilde{z}(0, t) \\
\widetilde{w}(L, t)
\end{array}\right], \\
\text { with } G_{2}=\left[\begin{array}{cc}
-\frac{1}{k_{v}} & 0 \\
1-\frac{a \rho^{*}}{v^{*}}-\frac{k_{\rho}}{v^{*} k_{v}} & -\frac{k_{\rho}}{v^{*}}
\end{array}\right] .
\end{gathered}
$$

By selecting the boundary feedback gains in (55) with $\left|k_{\rho}\right|<v^{*}$ and $\left|k_{v}\right|<1$, it holds $\rho\left(G_{1}\right)<1$ and $\rho\left(G_{2}\right)>$ 1 . Then the free-flow mode with the boundary condition matrix $G_{1}$ is exponentially stable in $L^{2}$-norm, and the congestion mode with $G_{2}$ is unstable (Coron et al. (2007)). While, the overall MJLH system (52) might be exponentially mean-square stable or not, in $L^{2}$-norm, under the Markov process $\sigma(t)$. 


\subsection{Simulations}

The developed boundary feedback strategy (55) and the stability conditions are now tested with numerical simulations based on the MJLH system (54) presented above. To this end, we consider a local freeway section whose road traffic parameters are given, respectively, as $\rho_{m}=$ 200 veh./hour, $v_{f}=160 \mathrm{~km} /$ hour, $a=0.8$, $p_{\text {in }}=6000$ veh./hour, $r_{0}=2000$ veh./hour, and the total road length is $1 \mathrm{~km}$, i.e., $x \in[0,1]$.

After clustering the historical traffic data, such as the speed-density records in Fig. 1, we represent the free-flow and the congestion modes with two typical traffic states $\left(\rho_{1}, v_{1}\right)=(25,120)$, and $\left(\rho_{2}, v_{2}\right)=(75,30)$, respectively. Then, the system matrices of the MJLH system (52) are given, respectively, as

$$
\Lambda_{1}=\left[\begin{array}{cc}
100 & 0 \\
0 & 120
\end{array}\right], \Lambda_{2}=\left[\begin{array}{cc}
-30 & 0 \\
0 & 30
\end{array}\right] \text {. }
$$

We consider the steady-state $\left(\rho^{*}, v^{*}\right)=(80,100)$ which satisfies the flux conservation condition (56), and choose the control gains in (55) as $k_{\rho}=20$ and $k_{v}=0.9$. The associated boundary condition matrices $G_{1}$ and $G_{2}$ with respect to the system matrices $\Lambda_{1}$ and $\Lambda_{2}$ are calculated, respectively, as

$$
G_{1}=\left[\begin{array}{cc}
-0.9 & 0 \\
-0.1240 & -0.2
\end{array}\right], G_{2}=\left[\begin{array}{cc}
-1.1111 & 0 \\
0.1378 & -0.2
\end{array}\right] .
$$

Firstly, we assume that the transition probability of two traffic modes follows the generator $\Pi_{1}=\left[\begin{array}{cc}-1 & 1 \\ 7 & -7\end{array}\right]$. Using (33)-(34) the upper and lower bounds of parameters $\mu_{1}$ and $\mu_{2}$ are computed as $\mu_{1}^{\pi}=-0.0042, \mu_{1}^{G}=0.1054$, $\mu_{2}^{\pi}=-0.1167$ and $\mu_{2}^{G}=-0.1054$, respectively. Solving conditions (30)-(31) of Corollary 1, we obtain $\mu_{1}=$ 0.0971, $\mu_{2}=-0.1072, P_{1}=\left[\begin{array}{cc}0.3107 & 0 \\ 0 & 0.0175\end{array}\right]$ and $P_{2}=$ $\left[\begin{array}{cc}4.6235 & 0 \\ 0 & 0.2101\end{array}\right]$. To numerically compute the solutions to the MJLH system (54), let us discretize them using a WENO scheme (Jiang and Shu (1996)) in Matlab. The initial deviations from the steady-state $\left(\rho^{*}, v^{*}\right)$ are given as $\widetilde{v}(x, 0)=4 \sin (0.5 \pi x)$ and $\widetilde{\rho}(x, 0)=5 \sin (0.5 \pi x)$, which satisfies the compatibility conditions and the initial mode is selected as $\sigma(0)=1$.

Fig. 3 depicts a path of Markov process with generator $\Pi_{1}$. Figs. 4 and 5 show the time-evolution of $v$ and $\rho$. It is observed that the traffic states clearly converge to their steady-state $\rho^{*}=80 \mathrm{veh} . / \mathrm{km}$ and $v_{1}^{*}=100 \mathrm{~km} /$ hour respectively as time increase, as expected from Corollary

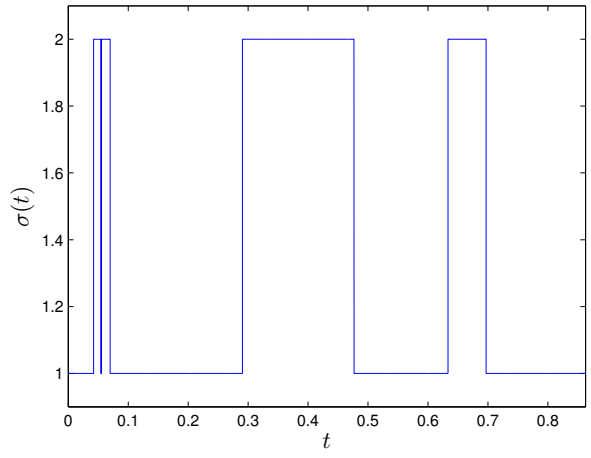

Figure 3. Simulation of a path of the Markov process with generator $\Pi_{1}$.

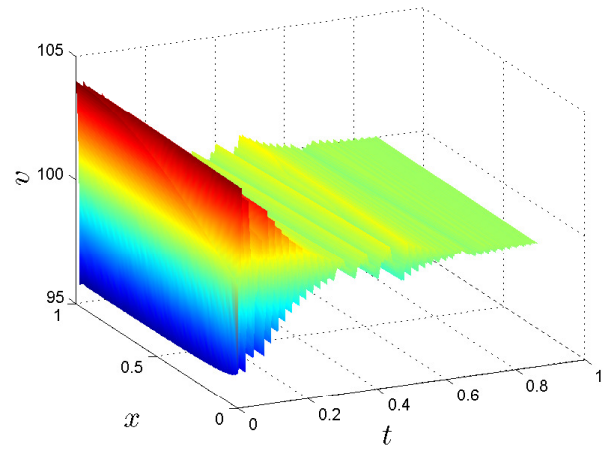

Figure 4. The evolution of $v$ of the MJLH traffic flow model with generator $\Pi_{1}$.

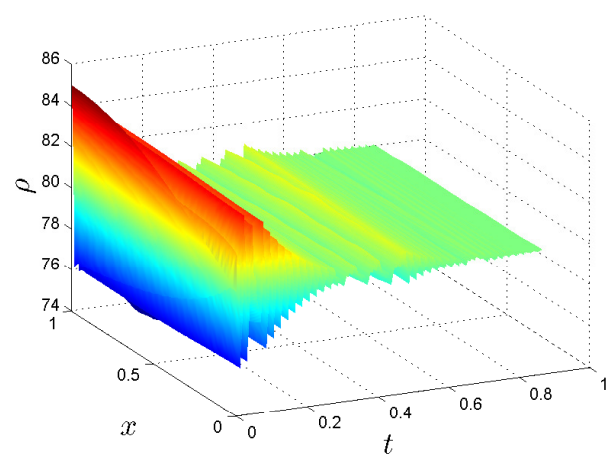

Figure 5. The evolution of $\rho$ of the MJLH traffic flow model with generator $\Pi_{1}$.

1. The simulation results illustrate an interesting phenomenon that a fixed boundary control, just using the traffic information from the downstream, might stabilize freeway traffic even though some congestion (unstable) modes occur occasionally.

In the other simulation, just only the Markov genera- 
tor is changed as $\Pi_{2}=\left[\begin{array}{cc}-1 & 1 \\ 4 & -4\end{array}\right]$. In this case, since $\mu_{2}^{\pi}=-0.0667$ and $\mu_{2}^{G}=-0.1054$, we have $\mu_{2}^{\pi}>\mu_{2}^{G}$. The conditions (30) and (31) of Corollary 1 do not hold. Figs. 6 depicts a path of the Markov process with generator $\Pi_{2}$. We can find that the sojourn time of the congestion mode has greatly increased. These factors finally overturn the balance between the boundary conditions $G_{1}, G_{2}$, and the transition probability $\Pi_{2}$. Figs. 7 and 8 show the unstable behaviors for a sample path of $v(x, t)$ and $\rho(x, t)$ under $\Pi_{2}$ using the same initial condition.

Also with the generator $\Pi_{2}$, after adjusting the proportional gains in control (55) as $k_{\rho}=10, k_{v}=0.95$, we can get a new set of the upper and lower bounds as $\mu_{1}^{\pi}=-0.0042, \mu_{1}^{G}=0.0513, \mu_{2}^{\pi}=-0.0667$ and $\mu_{2}^{G}=$ -0.0513 . In this case, conditions (30)-(31) of Corollary 1 hold and we compute $\mu_{1}=0.0473, \mu_{2}=-0.0553$, $P_{1}=\left[\begin{array}{cc}0.5261 & 0 \\ 0 & 0.0255\end{array}\right]$ and $P_{2}=\left[\begin{array}{cc}4.1475 & 0 \\ 0 & 0.1744\end{array}\right]$. Therefore, the MJLH traffic flow model (52) becomes exponentially mean-square stable again under the new boundary feedback control (55). For simplicity, we omit the figures of this case.

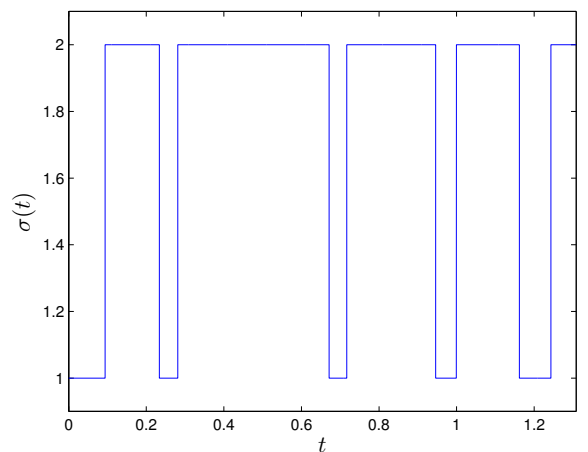

Figure 6. Simulation of a path of the Markov process with generator $\Pi_{2}$.

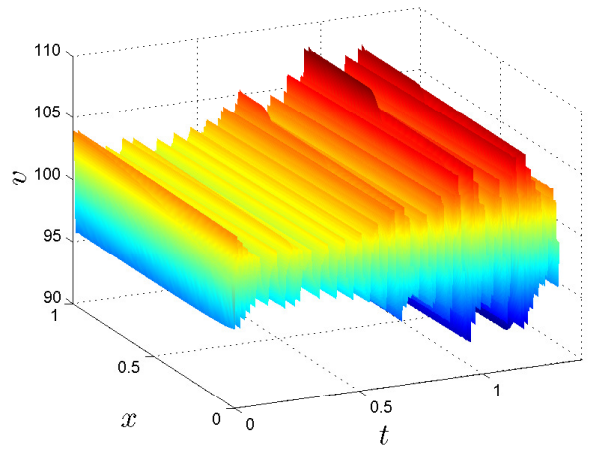

Figure 7. The evolution of $v$ of the MJLH traffic flow model with generator $\Pi_{2}$.

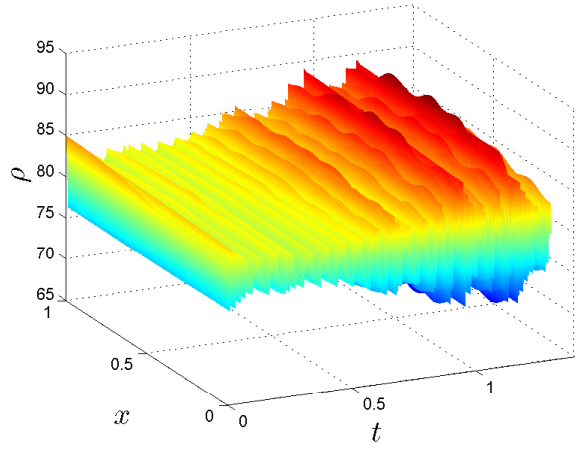

Figure 8. The evolution of $\rho$ of the MJLH traffic flow model with generator $\Pi_{2}$.

\section{Conclusion}

We present a class of switched systems, namely the MJLH system, in which the logical decision is Markov chain and subsystems are linear hyperbolic conservation laws. By means of a stochastic Lyapunov function, exponentially mean-square stability are derived dependeding on the balance between the boundary conditions and the transition probability. Theoretical contribution was applied to stabilize freeway traffic by integrating the onramp metering with the speed limit in the distributed control action. This work leaves many open questions. It is natural to extend theoretical results, such as Theorem 1 and Corollary 1 to more general MJLH systems with time-varying velocities. Some effort will also devoted to the study of more general MJLH systems governed by an unobservable Markov chain.

\section{References}

Amin, S., Hante, F. M., Bayen, A. M., 2012. Exponential stability of switched linear hyperbolic initialboundary value problems. IEEE Transactions on $\mathrm{Au}-$ tomatic Control 57 (2), 291-301.

Aw, A., Rascle, M., 2000. Resurrection of "second order" models of traffic flow. SIAM journal on applied mathematics 60 (3), 916-938.

Bastin, G., Coron, J.-M., 2016. Stability and Boundary Stabilization of 1-D Hyperbolic Systems. Progress in Nonlinear Differential Equations and Their Applications. Springer.

Boel, R., Mihaylova, L., 2006. A compositional stochastic model for real time freeway traffic simulation. Transportation Research Part B 40 (4), 319-334.

Boukas, E. K., 2005. Stochastic switching systems. Birkhauser, Basel, Berlin.

Colombo, R., 2003. Hyperbolic phase transitions in traffic flow. SIAM Journal on Applied Mathematics 63, 708-721.

Coron, J.-M., d'Andréa Novel, B., Bastin, G., 2007. A strict Lyapunov function for boundary control of hy- 
perbolic systems of conservation laws. IEEE Transactions on Automatic Control 52 (1), 2-11.

Costa, O. L. V., Fragoso, M. D., Todorov, M. G., 2013. Continuous-time Markov jump linear systems. Probability and Its Applications. Springer-Verlag, Berlin.

de Halleux, J., Prieur, C., Coron, J.-M., d'Andréa Novel, B., Bastin, G., 2003. Boundary feedback control in networks of open channels. Automatica 39, 13651376.

Fang, Y., Loparo, K. A., 2002. Stabilization of continuous-time jump linear systems. IEEE Transactions on Automatic Control 47 (10), 1590-1603.

Greenshields, B. D., 1935. A study of traffic capacity. In: Proceedings of the Highway Research Board. pp. 448-477.

Gugat, M., Dick, M., Leugering, G., 2011. Gas flow in fan-shaped networks: classical solutions and feedback stabilization. SIAM Journal on Control and Optimization 49 (5), 2101-2117.

Hante, F., Leugering, G., Seidman, T., 2009. Modeling and analysis of modal switching in networked transport systems. Applied Mathematics \& Optimization 59 (2), 275-292.

Haut, B., Bastin, G., Coron, J.-M., d'Andréa Novel, B., 2007. Lyapunov stability analysis of networks of scalar conservation laws. Networks and Heterogeneous Media 2 (4), 749-757.

Jiang, G. S., Shu, C. W., 1996. Efficient implementation of weighted ENO schemes. Journal of Computational Physics 126 (1), 202-228.

Kerner, B. S., 2009. Introduction to modern traffic flow theory and control. Springer Berlin Heidelberg.

Lamare, P.-O., Girard, A., Prieur, C., 2015. Switching rules for stabilization of linear systems of conservation laws. SIAM Journal on Control and Optimization $53(3), 1599-1624$.

Li, T.-T., 1994. Global classical solutions for quasilinear hyperbolic systems. Vol. 32 of RAM: Research in Applied Mathematics. Masson, Paris.

PeMS, 2004. Freeway Performance Measurement System. http://pems . berkeley.edu.

Prieur, C., Girard, A., Witrant, E., 2014. Stability of switched linear hyperbolic systems by Lyapunov techniques. IEEE Transactions on Automatic control 59 (8), 2196-2202.

Sumalee, A., Zhong, R. X., Pan, T. L., Szeto, W. Y., 2011. Stochastic cell transmission model (SCTM): A stochastic dynamic traffic model for traffic state surveillance and assignment. Transportation Research Part B 45 (2), 507-533.

Zhang, H. M., 2002. A non-equilibrium traffic model devoid of gas-like behavior. Transportation Research Part B 36 (3), 275-290.

Zhang, L., Mao, X., 2015. Vehicle density estimation of freeway traffic with unknown boundary demandsupply: an interacting multiple model approach. IET Control Theory and Application 9 (13), 1989-1995. 